\title{
The very high energy characteristics of shell-type SNRs at different ages.
}

\section{V.G. Sinitsyna*}

P.N. Lebedev Physical Institute, Leninsky pr. 53, Moscow, Russia

E-mail: sinits@sci.lebedev.ru

\section{V.Y. Sinitsyna}

P.N. Lebedev Physical Institute, Leninsky pr. 53, Moscow, Russia

E-mail: sinits@sci.lebedev.ru

\begin{abstract}
The investigation of VHE gamma-ray sources by any methods, including mirror Cherenkov telescopes, touches on the problem of the cosmic ray origin and, accordingly, the role of the Galaxy in their generation. The SHALON observations have yielded the results on Galactic supernova remnants (SNR) of different ages. Among them are: the shell-type SNRs Tycho's SNR (1572y), Cas A (1680y), IC 443 (age $\sim(3 \div 30) \times 10^{3}$ y), $\gamma$ Cygni SNR (age $\sim(5 \div 7) \times 10^{3}$ y) and classical nova GK Per (Nova 1901). For each of SNRs the observation results are presented with spectral energy distribution by SHALON in comparison with other experiment data and images by SHALON together with data from X-ray by Chandra and radio-data by CGPS. The collected experimental data have confirmed the prediction of the theory about the hadronic generation mechanism of very high energy $800 \mathrm{GeV}-100 \mathrm{TeV}$ gamma-rays in Tycho's SNR, Cas A and IC443. Recently, unique data on GK Per TeV gamma-ray emission were obtained with SHALON experiment. The X-ray data shows that, the nova remnant of GK Per could be a younger remnant that will resemble older SNRs (like IC 443) which interact with molecular clouds. GK Per is supposed to be a candidate for $\mathrm{TeV}$ gamma-ray emission due to the accelerated particles in the reverse shock region. The analysis of SHALON observation data revealed the main TeV-emission region coinciding with the position of central source of GK Per and the weak emission of shell, that is also observed in X-ray by Chandra.
\end{abstract}

The 34th International Cosmic Ray Conference,

30 July- 6 August, 2015

The Hague, The Netherlands

\footnotetext{
*Speaker.
} 


\section{Introduction}

The hypothesis that Supernova Remnants (SNRs) are unique candidates for cosmic-ray sources has been prevalent from the very beginning of cosmic-ray physics. The electron component of Cosmic Rays is well visible on the SNR emission through the wide range of electromagnetic spectrum from radio to low energy $\gamma$-ray emission, but the information on the nuclear Cosmic Ray component in SNRs can only be obtained from high and very high energy $\gamma$-ray observations. We present the observation results by SHALON telescope of shell type Galactic supernova remnants: Tycho's SNRs, Cas A, $\gamma$ Cygni SNR and IC 443 as well as the classical nova GK Per that is on the earliest SNR evolution stage. For each of SNRs the observation results are presented in Table 1 with spectrum parameters and in Fig. 1 with spectral energy distribution by SHALON ( $\triangle$ and $\mathbf{\Delta}$ ) in comparison with other experiment data and images by SHALON (color scale) in comparison with data from X-ray by Chandra (for GK Per, Cas A and Tycho) and radio-data by CGPS (for $\gamma$ CygniSNR and IC443). Also the theoretical predictions is shown.

\section{GK Per (Nova 1901)}

Nova Persei 1901 (GK Per) is one of the most extensively observed and studied classical nova shells over the entire electromagnetic spectrum. The X-ray shell around GK Per was first discovered with the ROSAT experiment and then it has been observed by Chandra telescope. The detection of the X-rays from the supernova remnant shell which are primarily due to bremsstrahlung of shock accelerated relativistic electrons, supposed the detection of $\gamma$-ray emission originated from $\pi^{\circ}$ - decay, secondary pp-interactions as well as possible contribution emission produced via Inverse Compton scattering (IC). Chandra X-ray data shows that, the nova remnant of GK Per could be a younger remnant that will resemble older SNRs like IC $443\left((3 \div 30) \times 10^{3}\right.$ year $)$ which interact with molecular clouds ([1] and refs. therein).

In accordance with program on long-term studies of metagalactic $\gamma$-ray sources, fifteen-yearlong observations of the central galaxy in the Perseus cluster, NGC 1275, are being carried out in the SHALON experiment [1, 2, 3, 4]. During the observations of NGC 1275 the SHALON field of

Table 1: The catalogue of galactic $\gamma$-ray sources by SHALON with parameters for spectrum fitting in form of power low with exponential cutoff $F(>E) \propto E^{k_{\gamma}} \times \exp \left(-E / E_{\text {cutoff }}\right)$.

\begin{tabular}{cccccc}
\hline Sources & Observable flux $^{a}$ & $k_{\gamma}$ & $E_{\text {cutoff }}, \mathrm{TeV}$ & Distance, kpc & Type \\
\hline Crab Nebula & $(2.12 \pm 0.12)$ & $-1.36 \pm 0.09$ & $19.0 \pm 2.0$ & 2.0 & PWN \\
Geminga & $(0.48 \pm 0.07)$ & $-0.39 \pm 0.05$ & $5.4 \pm 1.0$ & 0.25 & PSR or PWN \\
3C 58 & $(0.56 \pm 0.15)$ & $-1.33 \pm 0.12$ & - & 3.2 & PWN \\
Tycho's SNR & $(0.52 \pm 0.04)$ & $-0.93 \pm 0.09$ & $35.0 \pm 5.0$ & $3.1-3.3$ & Shell-type SNR \\
Cas A & $(0.64 \pm 0.10)$ & $-0.91 \pm 0.11$ & $10.3 \pm 2.5$ & 3.1 & Shell-type SNR \\
IC 443 & $(1.69 \pm 0.58)$ & $-1.94 \pm 0.16$ & - & 1.5 & Shell-type SNR \\
rCygni SNR & $(1.27 \pm 0.11)$ & $-0.95 \pm 0.09$ & $20.1 \pm 4.2$ & 1.5 & Shell-type SNR \\
GK Per & $(0.31 \pm 0.14)$ & $-1.90 \pm 0.36$ & - & 0.46 & Classical Nova \\
Cyg X-3 & $(0.68 \pm 0.04)$ & $-1.15 \pm 0.08$ & $75.0 \pm 10.2$ & 10.0 & HMX Binary \\
4U 2129+47 & $(0.19 \pm 0.06)$ & $-0.42 \pm 0.12$ & $10.0 \pm 3.0$ & 6.0 & LMX Binary \\
Her X-1 & $(0.45 \pm 0.18)$ & - & - & 6.6 & Binary \\
M57 & $(0.30 \pm 0.17)$ & - & - & 0.7 & Planetary nebula \\
\hline \multicolumn{7}{c}{ Integral flux at energy $>800 \mathrm{GeV}$ in units of $10^{-12} \mathrm{~cm}^{-2} \mathrm{~s}^{-1}$} &
\end{tabular}


view contains the source of nonthermal radio and X-ray emission GK Per (Nova 1901) of classical nova type as it located at $\sim 3^{\circ}$ North from NGC 1275 . GK Per as a source accompanying to NGC 1275 was observed with SHALON at the period from 1996y to 2012y for a total of 111 hours. The $\gamma$-ray source associated with the GK Per was detected above $2 \mathrm{TeV}$ by SHALON with a statistical significance $9.2 \sigma$ determined by Li\&Ma method. For the detection details see [1], table 1 and Fig. 1. The signal significance for this SNR is less then one for the source with similar flux and spectrum index obtained in the same observation hours because of less collection field of view relative to the standard procedure of SHALON experiment $[1,2,3,4]$. The corrections for the effective field of view were made to calculate source flux and energy spectrum. The analysis of $\gamma-$ ray shower arrival direction revealed the main $\mathrm{TeV}$-emission region coinciding with the position of central source of GK Per and the weak emission of shell, that is also observed in X-ray by Chandra (Fig. 1, right, [1] and refs. therein).

\section{Cas A supernova remnant (1680 year)}

Cas A is a youngest of historical SNR in our Galaxy. Its overall brightness across the electromagnetic spectrum makes it a unique object for studying high- and very high- energy phenomena in SNRs. Cas A was observed with SHALON telescope during the 68 hours in period of 2010 - 2013 yy. The $\gamma$-ray source associated with the SNR Cas A was detected above $800 \mathrm{GeV}$ (see table 1) with a statistical significance of $16.1 \sigma$ by Li\&Ma. Figure 1 right presents Chandra X-ray image of Cas A (lines) in comparison TeV structure in energy range of 0,8 - $30 \mathrm{TeV}$ by SHALON $([1,3]$ and refs therein). Cas A was detected in TeV $\gamma$-rays, by HEGRA, MAGIC, VERITAS and SHALON. The high energy $\gamma$ - ray emission from Cas A was detected with Fermi LAT in the range $500 \mathrm{MeV}-50 \mathrm{GeV}$. Solid lines show the very high energy gamma-ray spectra of hadronic origin. The leptonic model (IC and NB) predicts the TeV spectrum extends only up to energy about 10 $\mathrm{TeV}$. The detection of $\gamma$-ray emission at $5-30 \mathrm{TeV}$ by SHALON [3] and the hard spectrum below $1 \mathrm{TeV}$ would favor the $\pi^{\circ}$-decay origin of the $\gamma$-rays in Cas A ([1,3] and refs therein).

\section{Tycho's Supernova Remnant (1572 year)}

In observations of 1996 year a new galactic source was detected by SHALON in TeV energies (for details see [3]). This object was identified with Tycho's SNRs. Recently, Tycho's SNR was also confirmed with VERITAS in observations of 2008 - 2010 years. The $\gamma$-ray emission from Tycho's SNR was detected with Fermi LAT in the range $400 \mathrm{MeV}-100 \mathrm{GeV}$. The expected flux of $\gamma$-quanta from $\pi^{\circ}$-decay, extends up to $>30 \mathrm{TeV}$, while the flux of $\gamma$-rays originated from the IC scattering has a sharp cut-off above the few $\mathrm{TeV}$, so the detection of $\gamma$-rays with energies from 10 to $80 \mathrm{TeV}$ by SHALON (Fig. 1) is an evidence of their hadronic origin ([1,3] and refs therein).

$\gamma$ Cygni SNR (age $\sim(5 \div 7) \times 10^{3}$ years)

Since 1995, long-term observations of Cyg X-3 are being carried out in the SHALON. The $\gamma$-ray source associated with the Cyg X-3 was detected above $800 \mathrm{GeV}$ (see table 1, [4] and refs therein), Fig. 2, left) with a statistical significance of $35.3 \sigma$ (283.6 hours). A number of high activity period of Cyg X-3 were detected at $\mathrm{TeV}$ energies during the all observation time. The correlation soft $\mathrm{X}$-ray and $\mathrm{TeV}$ energy $\gamma$-ray fluxes is traced. To securely identify the detected emission with Cyg X-3, a timing analysis to search for the 4.8-hour orbital period of Cyg X-3 was performed (Fig. 2, right).

During the observations of Cyg X-3 the SHALON field of view contains $\gamma$ Cygni SNR as it located in Cygnus Region at $\sim 2^{\circ} \mathrm{SW}$ from Cyg X-3. So due to the large telescopic field of view 

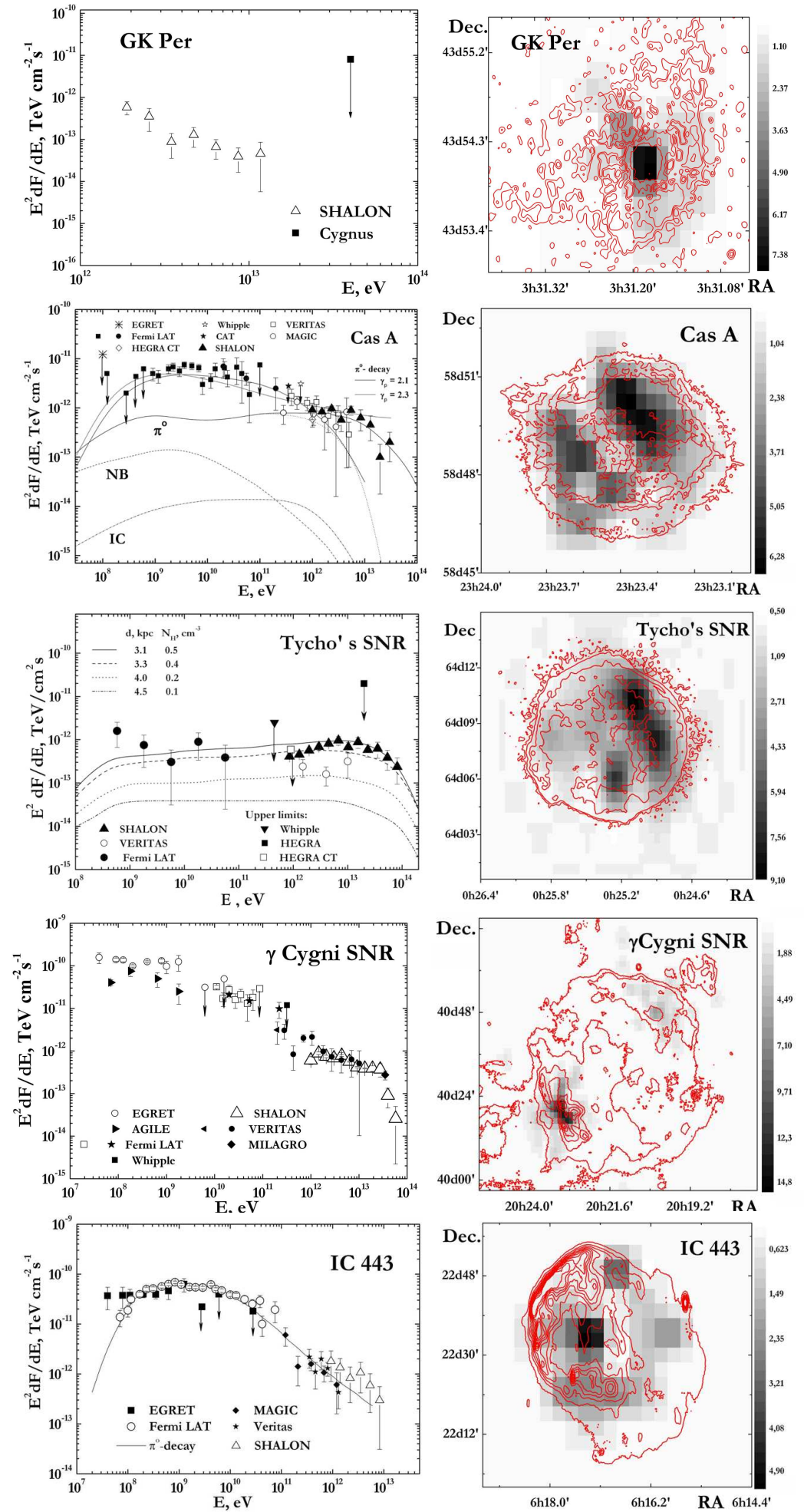

Figure 1: Spectral energy distributions (left) and images (right) of Supernova remnants by SHALON in comparison with other experiments (see text).

$\left(\sim 8^{\circ}\right)$ the observations of Cyg X-3 is naturally followed by the observations of this SNR. As a result, the $\gamma$-ray source located at $2^{\circ}$ from Cyg X-3 and associated with the $\gamma$ Cygni SNR was detected above $800 \mathrm{GeV}$ by SHALON with statistical significance of $14 \sigma[1,4]$. The analysis of 

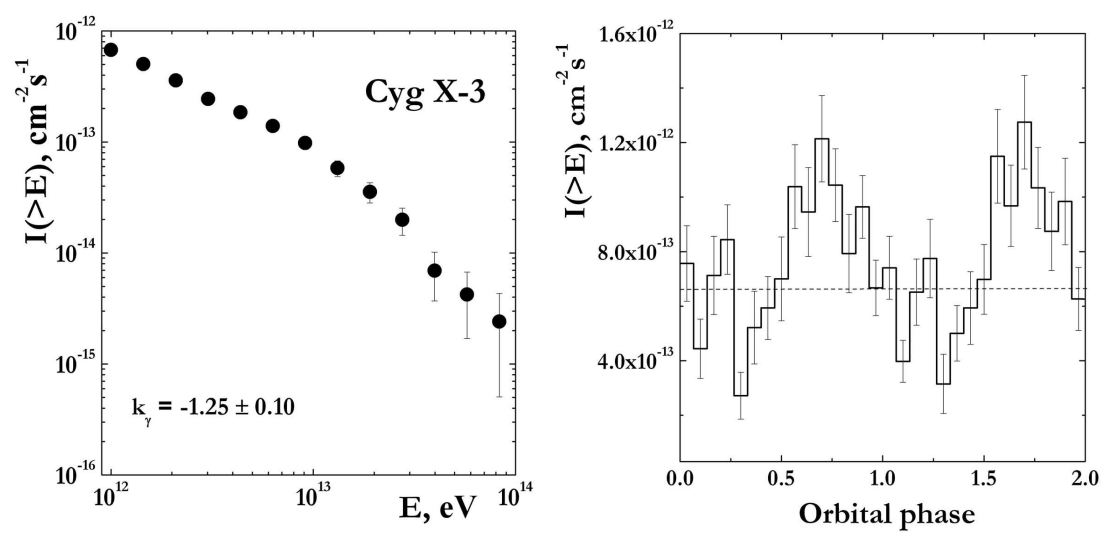

Figure 2: left: The integral energy spectrum of Cyg X-3; right: SHALON light curve of Cyg X-3 folded on the orbital period; The dashed line shows the level of averaged integral $\mathrm{TeV} \gamma$-ray flux (see table 1).

$\mathrm{TeV} \gamma$-ray arrival directions reveal two emission regions in $\gamma$ Cygni SNR: the main at the SouthEast part of SNR shell and second one at North. The main contribution of energy flux gives the SE region of SNR shell (see Fig. 1, right). Also, $\mathrm{TeV} \gamma$-ray emission regions correlate with the NW and SE parts of the shell visible in the radio energies by Canadian Galactic Plane Survey.

IC 443 supernova remnant (age $\sim(3 \div 30) \times 10^{3}$ years)

IC443 was detected by SHALON at energies from $800 \mathrm{GeV}$ up to $7 \mathrm{TeV}$ ([1] and refs therein) with statistical significance of $9.7 \sigma$. The favored scenario in which the $\gamma$-rays of $100 \mathrm{MeV}-7 \mathrm{TeV}$ energies are emitted in the shell of the IC443 SNR is $\pi^{\circ}$-decay which produced in the interactions of the cosmic rays with the interstellar gas. IC scattering can not explain the observed IC $443 \gamma$-ray emission as there is no bright source of seed photons in the region of the IC 443. The image of IC443 at TeV energies by SHALON is presented in Fig. 1, right in comparison with radio structure by CGPS (see lines). The analysis of arrival directions of $\gamma$-rays with energies $800 \mathrm{GeV}-7 \mathrm{TeV}$ reveal the correlation of $\mathrm{TeV} \gamma$-ray emission maxima with $\mathrm{MeV}-\mathrm{GeV}$ emission observed by Fermi LAT, also TeV $\gamma$-ray emission of South and SW parts of IC 443 shell correlated with the position of swept out dense molecular cloud.

\section{Conclusion}

The observation results of Galactic shell-types supernova remnants on different evolution stages GK Per (Nova 1901), Cas A, Tycho's SNR, $\gamma$ Cygni SNR and IC 443 by SHALON mirror Cherenkov telescope are presented. The TeV $\gamma$-ray emission of classical nova GK Per, that could be a shell-type supernova remnant on early evolution stage, was detected for the first time by SHALON. Also, very high energy $\gamma$-rays from the shell of GK Per, visible in the X-rays, were detected with SHALON experiment for the first time. The experimental data have confirmed the prediction of the theory about the hadronic generation mechanism of very high energy $\gamma$-rays in Tycho's SNR, Cas A and IC443.

\section{References}

[1] V.G. Sinitsyna, V. Y. Sinitsyna, Bulletin of the Lebedev Physics Institute, 42(6), 200 (2015).

[2] V.G. Sinitsyna, V. Y. Sinitsyna, Astronomy Letters 40(2-3), 91 (2014).

[3] V.G. Sinitsyna, V. Y. Sinitsyna, Astronomy Letters 37(9), 621 (2011).

[4] V.G. Sinitsyna, V. Y. Sinitsyna, Bulletin of the Lebedev Physics Institute, 40(5), 113 (2013). 